\title{
ESPORTES DE AVENTURA VERSUS EXERCÍCIOS E ESPORTES: CONSIDERAÇÕES SOBRE AS REGULAÇÕES MOTIVACIONAIS DE ADOLESCENTES
}

\author{
Thiago Sousa Matias
}

Universidade Federal de Santa Catarina, Florianópolis, Santa Catarina, Brasil

\author{
Alexandro Andrade \\ Universidade do Estado de Santa Catarina, Florianópolis, Santa Catarina, Brasil \\ Julia Morais Manfrin \\ Universidade Federal de Santa Catarina, Florianópolis, Santa Catarina, Brasil
}

\begin{abstract}
Resumo
Objetivou-se neste estudo comparar as motivações de praticantes de esporte de aventura a outras modalidades e verificar se as motivações predizem as escolhas de atividade física. Trata-se de um estudo transversal com 2.757 adolescentes. A regulação externa é menor nos esportes de aventura $(0,52 \pm 0,75)$, comparada a outras modalidades $(0,71 \pm 0,86)$. A regulação identificada (aventura 2,44 $\pm 1,09$ - outras modalidades 2,21 $\pm 1,08 ; \mathrm{p}=0,035$ ) e a regulação intrínseca (aventura 2,95 $\pm 0,99$ - outras modalidades 2,56 $\pm 1,16 ; \mathrm{p}=0,01$ ) são maiores para os esportes de aventura. A regulação intrínseca está associada à escolha por esportes de aventura $(\mathrm{OR}=0,71$; IC 95\% = 0,54-0,93). Adolescentes que escolhem esportes de aventura são mais autodeterminados, favorecendo a manutenção da atividade física no lazer.
\end{abstract}

Palavras-chave: Atividade motora. Motivação. Psicologia do Esporte. Estudantes.

\section{THE ROLE OF SELF-DETERMINATION THEORY ON ADOLESCENT CHOICES: ADVENTURE SPORTS VERSUS OTHERS PHYSICAL ACTIVITIES}

\begin{abstract}
This study aimed to verify motivational differences and associations between adventure sport, and other physical activities choices. A cross-sectional population-based study of 2,757 adolescent students from south of Brazil. External regulation was lower in adventure sports $(0,52 \pm 0,75)$ compared to others $(0,71 \pm 0,86)$. However, identified regulation (adventure $2,44 \pm 1,09$ - others $2,21 \pm 1,08 ; \mathrm{p}=0,035$ ), and intrinsic regulation (adventure 2,95 $\pm 0,99$ - others $2,56 \pm 1,16 ; \mathrm{p}=0,01)$ was higher in adventure sports. Intrinsic regulation was associated to adventure sport in leisure-time (OR $=0,71$; IC 95\% $=0,54-0,93)$. We confirm the important link between autonomous motivation and adventure sports. Adventure sports may be important for physical activity maintenance in young people.
\end{abstract}

Keywords: Motor Activity. Motivation. Psychology, Sports. Students. 


\section{DEPORTES DE AVENTURA VERSUS EJERCICIOS Y DEPORTES: CONSIDERA- CIONES SOBRE LAS REGULACIONES MOTIVACIONALES DE ADOLESCENTES}

\section{Resumen}

Este estudio objetivó comparar y asociar las motivaciones de practicantes de deporte de aventura a otros deportes. Estudio transversal de base poblacional con 2.757 adolescentes de la gran Florianópolis. La regulación externa es menor $(0,52 \pm 0,75)$ para los deportes de aventura comparado a otras modalidades $(0,71 \pm 0,86)$. La regulación identificada (aventura $2,44 \pm$ 1,09 - otras modalidades $2,21 \pm 1,08 ; \mathrm{p}=0,035$ ) y regulación intrínseca (aventura 2,95 $\pm 0,99$ - otras modalidades $2,56 \pm 1,16 ; p=0,01)$ es mayor para el deporte de aventura. La regulación intrínseca está asociada a la elección por deportes de aventura en el ocio $(\mathrm{OR}=0,71$, IC $95 \%=0,54-0,93)$. Los deportistas de deportes de aventura son más autodeterminados y los deportes de aventura pueden favorecer la iniciación y el mantenimiento de la actividad física.

Palabras clave: Actividad Motora. Motivación. Psicología del Deporte. Estudiantes.

\section{Introdução}

As escolhas de adolescentes na prática de atividade física (AF) no lazer perpassam por diferentes motivações, estas vão das mais reguladas externamente até escolhas mais intrinsecamente motivadas (SILVA et al., 2012). Há evidências que algumas modalidades podem naturalmente representar processos motivacionais mais internos, como é o caso dos esportes de aventura (AMARAL; DIAS, 2008).

O esporte de aventura é uma demanda crescente e perfaz a escolha de alguns adolescentes e jovens que buscam na aventura outros sentidos e significados emocionais na prática esportiva (VIEIRA et al., 2011). A aventura está relacionada com a busca de desafios, no qual prevalece a imprevisibilidade (SPINK; ARAGAKI; ALVES, 2005). Ainda nesse contexto imprevisível, essas atividades parecem se relacionar com questões afetivo-emocionais como estados de medo, de risco, de prazer e ansiedade (LAVOURA; SCHWARTZ; MACHADO, 2008; VIEIRA et al., 2011; MORAES; OLIVEIRA, 2006).

Diferente de alguns esportes, os esportes de aventura parecem mais complexos de entender quando são observadas as razões e as motivações para praticá-lo (KERR; MACKENZIE, 2012). Ao que parece, as motivações ultrapassam os condicionantes externos como os relacionados ao consumo ou ao modismo; para essas modalidades, são sugeridas que as respostas afetivas mais intrínsecas como a relação com a natureza, o autoconhecimento e o conhecimento do outro influenciam as escolhas por essa atividade (LAVOURA; SCHWARTZ; MACHADO, 2008).

No contexto da motivação, alguns pressupostos teóricos têm observado que as razões para a adoção de determinados comportamentos variam conforme o grau de autonomia que o indivíduo tem ou o grau em que ele é menos ou mais autodeterminado (DECI; RYAN, 1985). Essas motivações estão relacionadas ao conjunto de regulações que leva o indivíduo a adotar, manter ou abandonar a prática (DUMITH; DOMINGUES; GIGANTE, 2008). Estudos têm observado que algumas modalidades apresentam características que levam os indivíduos a maiores sensações de prazer e satisfação, como parece ser o caso dos esportes de aventura (AMARAL; DIAS, 2008). Neste caso, o lócus motivacional ligado a essas características indicam motivações mais intrínsecas e consequentemente mais autodeterminadas para a prática de AF (SILVA et al., 2012).

Há evidências de que são as motivações mais intrínsecas que prevalecem sobre os esportes de aventura, visto que as regulações mais internas geram maior sensação de prazer, autossatisfação, recompensa pelo esforço, alívio e serenidade diante dos riscos oferecidos 
pelos esportes de aventura (MORAES; OLIVEIRA, 2006). Em um estudo com praticantes de escalada e skate downhill, foi observado que o tipo de motivação mais presente foi a intrínseca, tanto para atingir os objetivos quanto para estimular e significar as experiências com a prática (VIEIRA et al., 2011).

Todavia, apesar dos indicativos de motivações mais intrínsecas para os esportes de aventura, são poucas as evidências que permitem inferir sobre as diferenças entre praticantes de esportes de aventura e de outras modalidades. Os esportes de aventura têm sido investigados em contextos formais de prática como o esporte competitivo (LAVOURA; MACHADO, 2006; LAVOURA; BOTURA; MACHADO, 2006; MORAES; OLIVEIRA, 2006), não sendo possível inferir os resultados para o contexto do lazer. Nesse caso, emergem algumas problemáticas de pesquisa como: existem diferenças nas regulações motivacionais de adolescentes praticantes de esportes de aventura no lazer comparadas a outras modalidades de AF e esportes no lazer? As regulações motivacionais predizem as escolhas de AF no lazer de adolescentes?

Evidencia-se aqui o estudo das motivações, ao perceber que os esportes de aventura podem representar uma oportunidade educacional; observa-se que a prática promove o autoconhecimento, o respeito pela natureza e a capacidade de transpor desafios, sentimentos essenciais para a formação do ser humano (PEREIRA; ARMBRUST; RICARDO, 2008). Como atividade de lazer, ao pressupor motivações mais intrínsecas, pode favorecer a iniciação e a manutenção de crianças e adolescentes em atividades físicas e esportes (LAVOURA; SCHWARTZ; MACHADO, 2008). Sendo assim, o objetivo deste estudo foi (a) comparar as motivações de adolescentes praticantes de esporte de aventura a outras atividades físicas e esportes e (b) verificar se as regulações motivacionais predizem as escolhas de AF no lazer de adolescentes.

\section{Métodos}

Este estudo caracteriza-se como de delineamento transversal e de base populacional. Trata-se de análise secundária do projeto Educação Física e Esporte em Santa Catarina: Pesquisa e Desenvolvimento nas Escolas Públicas, iniciado em 2014. Foram elegíveis para o estudo estudantes de escolas públicas do $9^{\circ}$ ano do ensino fundamental ao $3^{\circ}$ do ensino médio, de ambos os sexos, estudantes do período matutino e vespertino. Participaram do estudo 2.757 estudantes adolescentes (1.061 meninos e 1.224 meninas), com média de idade de $15 \pm 4$ anos.

Para o cálculo amostral, foi considerado um erro máximo de 3 pontos percentuais e confiabilidade de $95 \%$. A prevalência foi estimada em $50 \%$ e, levando em conta os conglomerados escolares, foi considerado um efeito do desenho de 1,5 (incremento de $50 \%$ na amostra). Uma correção foi aplicada ponderando os possíveis erros de preenchimento e questionários incompletos (40\%). O tamanho amostral requerido foi de 2.243 estudantes. A amostra foi derivada de 26 diferentes escolas públicas de oito cidades da grande Florianópolis (SC. Brasil).

Os estudantes participaram do estudo mediante entrega do Termo de Consentimento Livre e Esclarecido, devidamente assinado pelos pais ou responsáveis, quando menores de 18 anos de idade. A pesquisa foi autorizada pelo Comitê de Ética em Pesquisa em Seres Humanos (CEP) da Universidade do Estado de Santa Catarina (protocolo $\mathrm{n}^{\circ}$ 502.531).

As escolhas de AF foram analisadas através do Questionário de Atividade Física Habitual (FLORINDO et al., 2006). O questionário prioriza em sua avaliação os exercícios físicos e esportes e tem 17 questões, divididas em dois blocos: 1 - esportes ou exercícios físicos (15 questões) e 2 - atividades físicas de locomoção para a escola (2 questões). Ele avalia a AF semanal dos blocos 1 e 2 e anual do bloco 1 . O questionário foi padronizado para gerar esco- 
res das atividades físicas em minutos/ano e minuto/semana. Por exemplo, no bloco 1, a partir da modalidade indicada, multiplica-se a duração diária em minutos pela frequência semanal e pelos meses por ano de prática (minutos por dia x frequência semanal x 4 x meses por ano). Até três modalidades são indicadas e, nesta pesquisa, foram utilizadas apenas as informações qualitativas do bloco 1 para identificar as escolhas de AF e esportes. As diferentes escolhas foram organizadas em dois grupos: (0) esportes de aventura e (1) AF e outros esportes. Esportes de aventura são atividades designadas por maior grau de risco, considerando condições de imprevisibilidade e de adequação às condições de altura, velocidade, equilíbrio ou outras variantes não plenamente controladas pelo praticante. São, em muitos casos, praticados na natureza como surf, escalada, slackline, entre outros. AF e outros esportes incluem, por exemplo, treinamento de força, treinamento aeróbio, atividades em academia, caminhada, entre outras; esportes incluem as atividades orientadas por regras e objetivos praticados em ambientes específicos.

O processo de agrupamento foi baseado no estudo de Frederick e Ryan (1993). Nesse sentido, dois pesquisadores foram solicitados a agrupar as atividades reportadas pelos estudantes nos grupos que elas melhor representam. Diferente de Frederick e Ryan, que usaram $Q$-sort technique e identificaram quatro diferentes grupos, neste estudo, os dois grupos foram definidos previamente. Atividades conduzidas no contexto da Educação Física escolar não foram consideradas. Para testar a reprodutibilidade da categorização inicial proposta, outros dois pesquisadores foram requeridos a realizarem o mesmo processo. Para cada categoria, o percentual de concordância foi de $91 \%$ para os esportes de aventura e $98 \%$ para as outras atividades.

Para a avaliação da motivação, foi utilizado o Questionário de Regulação do Comportamento para o Exercício Físico - 2 (MARKLAND; TOBIN, 2004). Tal questionário se baseia na TAD e tem o objetivo de quantificar as diferentes regulações motivacionais, internas e externas, bem como a amotivação, relacionadas à prática de exercícios físicos. Este questionário é composto por 19 subescalas do tipo Likert, com cinco opções de resposta de 0 (Não é verdade para mim) a 4 (Muitas vezes é verdade para mim), separadas em cinco diferentes construtos: amotivação, regulação externa, regulação introjetada, regulação identificada e regulação intrínseca.

A medida do estrato socioeconômico foi obtida por meio da classificação proposta pela Associação Brasileira de Empresas em Pesquisa (ABEP). A classificação utiliza de informações quanto aos itens existentes na residência do participante e da escolaridade do chefe da família.

As análises estatísticas foram conduzidas no pacote estatístico SPSS para Windows 17.0. O tratamento dos dados ocorreu em duas etapas. Na primeira, foi realizada estatística descritiva, visando verificar as medidas de tendência central, frequências e percentuais, bem como as medidas de variabilidade. Na segunda etapa foi conduzida estatística inferencial. Para comparação dos grupos de AF foi utilizado o teste $t$ de student seguido do tamanho de efeito (Cohen's d). As associações entre as diferentes regulações motivacionais e as escolhas de AF no lazer (esportes de aventura (0) vs. outras modalidades (1)) foram avaliadas por meio de modelos de regressão logística binária bruta e ajustadas, dos quais se obtiveram os valores de odds ratio (OR) e intervalos de confiança de 95\% (IC95\%). Adotou-se a entrada simultânea das variáveis no modelo ajustado, com ajuste para as variáveis sociodemográficas sexo, idade e estrato socioeconômico.

\section{Resultados}

A opção por esportes de aventura representa $4,1 \%$ da amostra. Foi observado que a regulação externa é significativamente menor $(0,52 \pm 0,75)$ para os praticantes de esporte de a- 
ventura comparado a outras modalidades $(0,71 \pm 0,86), p=0,03$; Cohen's $d=0,23$. Contrariamente, a regulação identificada (esportes de aventura 2,44 $\pm 1,09$ - outras modalidades 2,21 $\pm 1,08$; $\mathrm{p}=0,035$; Cohen's d=0,21) e motivação intrínseca (esportes de aventura 2,95 $\pm 0,99$ - outras modalidades 2,56 $\pm 1,16 ; \mathrm{p}=0,01$; Cohen's $\mathrm{d}=0,36$ ) é significativamente maior no grupo dos adolescentes praticantes de esportes de aventura comparada às outras modalidades (Figura 1).

Figura 1. Comparação das regulações motivacionais de praticantes de esportes de aventura a outras modalidades de exercício e esportes (média), Florianópolis - SC, 2014 Reg. $=$ regulação; $*$ = diferença significativa para $\mathrm{p}<0,05$.

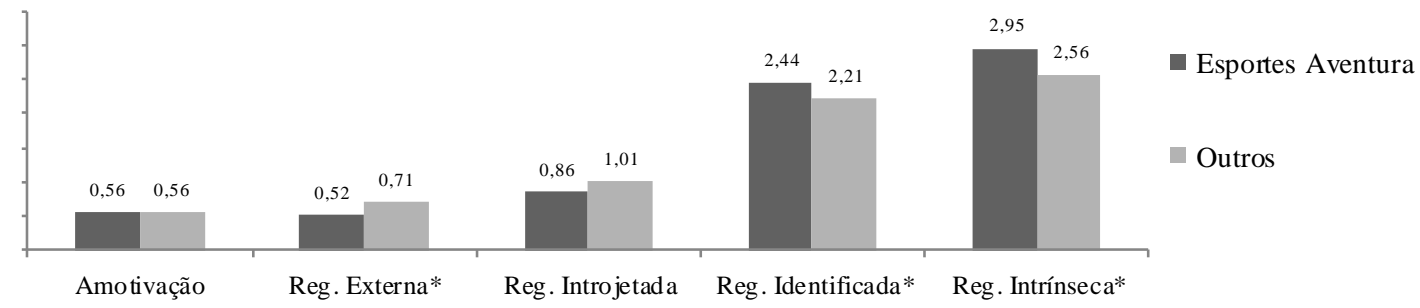

Fonte: Elaborada pelos autores, 2017.

Quando analisada a associação das diferentes regulações motivacionais com as escolhas de AF no lazer (Tabela 1), foi observado na análise ajustada que a regulação intrínseca está associada à escolha por esportes de aventura no lazer $(\mathrm{OR}=0,71 ; \mathrm{IC} 95 \%=0,54-0,93)$.

Tabela 1. Associação entre regulações motivacionais e escolhas de AF de estudantes adolescentes, Florianópolis - SC, 2014

\begin{tabular}{lcc}
\hline & $\begin{array}{c}\text { Bruta } \\
\text { OR (IC 95\%) }\end{array}$ & $\begin{array}{c}\text { Ajustada } \\
\text { OR (IC 95\%) }\end{array}$ \\
\hline Reg. Motivacionais & & \\
Amotivação & $0,99(0,79-1,27)$ & $0,81(0,61-1,07)$ \\
Reg. Externa & $1,35(1,03-1,78)$ & $1,35(0,97-1,86)$ \\
Reg. Introjetada & $1,14(0,94-1,40)$ & $1,22(0,96-1,54)$ \\
Reg. Identificada & $0,82(0,68-0,99)$ & $1,02(0,77-1,36)$ \\
Reg. Intrínseca & $0,72(0,60-0,87)$ & $\mathbf{0 , 7 1}(\mathbf{0 , 5 4 - 0 , 9 3 )}$ \\
\hline
\end{tabular}

Reg. = regulação; OR = odds ratio; $\mathrm{IC}=$ intervalo de confiança.

Análises ajustadas para sexo, idade e estrato socioeconômico.

Fonte: Elaborada pelos autores, 2017.

\section{Discussão}

Este estudo investigou as regulações motivacionais de adolescentes no contexto dos esportes de aventura. Foi observado que as regulações motivacionais de praticantes de esportes de aventura comparadas a outras práticas de AF e esportes são diferentes. Praticantes de esportes de aventura são menos regulados externamente e mais regulados internamente (regulação identificada e intrínseca), o que indica lócus motivacional mais prazeroso e satisfatório para a escolha do adolescente.

O conjunto de motivações também foi investigado em outros estudos no contexto dos esportes de aventura, e as evidências dão suporte para a relação positiva entre motivações 
intrínsecas e esportes de aventura (MORAES; OLIVEIRA, 2006; LAVOURA; MACHADO, 2006; VIEIRA et al., 2011). No estudo de Vieira et al. (2011), foram analisados as regulações motivacionais e estado de fluxo de praticantes de escalada e skate downhill e foi observado que a regulação intrínseca caracteriza a prática desses esportes. Ainda, foram observados valores altos para a motivação introjetada e identificada, que é um tipo de motivação externa, mas que apresenta alto grau de internalização.

Também, em um estudo com praticantes de alpinismo de alto nível, observou-se que os escaladores, em meio a dificuldades e imprevisibilidades, devem manter altos seus níveis motivacionais, e esses níveis motivacionais sugerem as regulações mais internas, uma vez que as emoções mais relatadas pelo estudo foram: autorrealização, prazer, solidariedade, liberdade e recompensa pelo esforço, sentimentos esses associados a níveis elevados de motivação intrínseca (MORAES; OLIVEIRA, 2006). Esses achados corroboram os nossos e indicam que a prática e a manutenção dos esportes de aventura são dependentes do conjunto de motivações, principalmente as mais internas, como a regulação identificada e intrínseca.

Ainda, nosso estudo observou que a regulação intrínseca prediz a escolha de adolescentes por esportes de aventura no lazer, e a literatura sugere que as emoções mais internas, característica dos esportes de aventura, implicam a escolha e a manutenção dos esportes de aventura (AMARAL; DIAS, 2008; LAVOURA; SCHWARTZ; MACHADO, 2008; KERR; MACKENZIE, 2012).

Em um estudo qualitativo com iniciantes na prática de rapel, foi observado que as emoções mais expressadas pelos praticantes tinham características motivacionais mais internas, o que foi chamado de "sensações prazerosas", tais como: experimentação, libertação e realização. Emoções essas que, segundo os autores, favorecem a reaproximação dos praticantes com a natureza, com as suas emoções e o reaprendizado do respeito com outros seres (LAVOURA; SCHWARTZ; MACHADO, 2008). Ainda, essas emoções também foram mencionadas em um estudo com praticantes de surf, sendo esses mesmos sentimentos antecipados pelos praticantes antes de iniciarem na prática, o que os fizeram aderir ao esporte de aventura e nele permanecer (AMARAL; DIAS, 2008). Esses achados estão relacionados com as repercussões emocionais da prática de esportes de aventura e podem, por conseguinte, justificar as expectativas iniciais dos praticantes como o anseio ao prazer, à aventura e a liberdade.

Um estudo realizado nos Estados Unidos aponta os diversos motivos que as pessoas encontram para praticar esportes de aventura, como: busca pela autonomia, relações de vínculo com o ambiente e com as pessoas, além da busca pela superação de desafios (KERR; MACKENZIE, 2012). Esses conjuntos de evidências corroboram o pressuposto teórico deste estudo e estão em conformidade com ele. A Teoria da Autodeterminação sinaliza que motivações mais autodeterminadas estão associadas às escolhas e à manutenção de determinadas práticas de AF (DECI; RYAN, 2000).

Adolescentes mais motivados intrinsicamente tendem a optar pela prática de esportes de aventura, pois estes estão relacionados à convivência com a imprevisibilidade, com o desconhecido e isso se conecta com a busca por significados e experiências emocionais, diferenciando-se de outras práticas de AF e esportes (PEREIRA; ARMBRUST; RICARDO, 2008). Nos esportes de aventura, à medida que aumenta a percepção da capacidade do praticante, também aumenta a percepção da dificuldade, e isso traz sensações de prazer e satisfação aos indivíduos (VIEIRA et al., 2011).

O prazer e a satisfação, conforme evidenciados na TAD (DECI; RYAN, 1985), são os principais lócus de causalidade para motivações mais internas. Essas causalidades estão associadas ao suprimento de necessidades psicológicas básicas, como a competência, o vínculo e a autonomia (VIERLING; STANDAGE; TREASURE, 2007). Dessa forma, esses adolescentes, com suas necessidades psicológicas básicas supridas, podem estar mais dispostos a encarar os 
desafios e as imprevisibilidades inerentes aos esportes de aventura (MORAES; OLIVEIRA, 2006).

Matias (2016) aponta a importância de entender as repercussões emocionais das diferentes escolhas de AF no lazer. Nesse sentido, sinaliza que os estudos no contexto da AF e a promoção da saúde vêm excluindo variáveis interpessoais, sociais e psicológicas importantes para a escolha e a manutenção da AF de lazer; essas relações são mais positivas quando o comportamento está associado às consequências afetivas e à antecipação positiva da resposta afetiva.

O esporte de aventura pode ser uma boa opção na promoção da AF de lazer na população adolescente, pois, quanto mais motivados intrinsecamente, maiores as chances de permanecerem no comportamento frente à AF (MATIAS et al., 2014). No âmbito da Educação Física escolar, e considerando a dificuldade de motivar adolescentes nas aulas, o esporte de aventura pode-se apresentar como uma atividade pedagogicamente inovadora, ao promover respostas emocionais ligadas ao prazer, à superação de desafios, à criação de vínculos, à liberdade e à satisfação na prática (MORAES; OLIVEIRA, 2006; LAVOURA; SCHWARTZ; MACHADO, 2008; AMARAL; DIAS, 2008; MARINHO, 2008; KERR; MACKENZIE, 2012).

São apresentadas algumas limitações importantes neste estudo. Os esportes de aventura são pouco estudados no contexto das variáveis psicológicas e são, na sua grande maioria, analisados no contexto formal/competitivo, o que dificulta a comparação com outras pesquisas. É importante considerar também que, apesar de avaliar uma relação unidirecional entre as variáveis investigadas, é plausível que a motivação tenha relação bidirecional ou faça a mediação das relações que se estabelecem com as escolhas de AF.

Apesar das limitações apresentadas, nossas evidências parecem mostrar-se consistentes, ao confrontar os esportes de aventura com outras escolhas de esportes e AF. Nossos achados sinalizam motivações mais internas para os esportes de aventura também no contexto do lazer de adolescentes, sendo que a maior parte dos estudos é com adultos e no contexto formal/competitivo. Ainda que transversal, trata-se de um estudo de base populacional e com delimitação teórica clara e previamente delimitada.

\section{Considerações finais}

Foi observado que adolescentes praticantes de esportes de aventura mostraram-se mais autodeterminados, comparados aos praticantes de outras modalidades de AF e esportes. As motivações intrínsecas para a prática de AF estiveram associadas à escolha por esportes de aventura na adolescência. São observados que processos intrinsecamente motivados implicam maior prazer e satisfação com a prática de AF e são condições psicológicas importantes para a adoção e a manutenção da prática de comportamentos fisicamente ativos. Nossos resultados confirmam a hipótese de que as motivações mais internas estão associadas aos antecedentes afetivo-emocionais característicos dos esportes de aventura, como prazer e satisfação. Sugerese que algumas modalidades, como os esportes de aventura, podem naturalmente se apresentar mais intrinsecamente motivantes para os jovens.

Estes achados são potencialmente importantes quando são observados os desafios e as dificuldades na promoção de AF na adolescência. Os interesses dos jovens na adoção de determinados comportamento são, em muitos casos, volúveis e influenciados por condicionantes sociais como os modismos e as questões estéticas. Por isso, ao observar a associação entre esporte de aventura e motivações internas, são sinalizadas possibilidades de prática de AF na adolescência em que essas dificuldades são amenizadas ou menos condicionadas por motivações externas. Sugere-se que os esportes de aventura possam favorecer a iniciação e a manutenção de adolescentes em atividades físicas e esportivas. 


\section{Referências}

AMARAL, A. V. de; DIAS, C. A. G.. Da praia para o mar: motivos à adesão e à prática do surfe. Licere, Belo Horizonte, v. 11, n. 3, p. 1-22, dez. 2008.

DECI, E., \& RYAN, R. The "what" and "why" of goal pursuits: human needs and the self determination of behavior. Psychological Inquiry, v. 11, n. 4, p. 227-268, 2000.

DECI, E.; RYAN, R. Intrinsic motivation and self-determination in human behavior. New York: Plenum, 1985.

DIAS, C. A. G. Por um programa investigativo para os esportes na natureza. Licere, Belo Horizonte, v. 11, n. 1, p.1-21, abr. 2008.

DUMITH, S. C.; DOMINGUES, M. R.; GIGANTE, D. P. Estágios de mudança de comportamento para a prática de atividade física: uma revisão de literatura. Revista Brasileira de Cineantropometria e Desempenho Humano, Florianópolis, v. 10, n. 3, p. 301-307, 2008.

FLORINDO, A. A. et al. Development and validationof a physical activity assessment questionnaire for adolescents. Revista de Saúde Pública, v. 40, n. 5, p. 802-809, 2006.

FREDERICK, C. M.; RYAN, R. M. Differences in motivation for sport and exercise and their relations with participation and mental health. Journal of sport behavior, Mobile, v. 16, n. 3 , p. 124, 1993.

KERR, J. H.; MACKENZIE, S. H. Multiple motives for participating in adventure sports. Psychology of Sport and Exercise, [s.1.], v. 13, n. 5, p.649-657, set. 2012.

LAVOURA, T. N.; BOTURA, H. M. L.; MACHADO, A. A. Estudo da ansiedade e as diferenças entre os gêneros em um esporte de aventura competitivo. Revista Brasileira de Educação Física, Esporte, Lazer e Dança, Santo André, v. 1, n. 3, p.74-81, set. 2006.

LAVOURA, T. N.; MACHADO, A. A. Esporte de aventura de rendimento e estados emocionais: relações entre ansiedade, autoconfiança e auto-eficácia. Motriz, Rio Claro, v. 12, n. 2, p.143-148, maio 2006.

LAVOURA, T. N.; SCHWARTZ, G. M.; MACHADO, A. A. Aspectos emocionais da prática de atividades de aventura na natureza: a (re)educação dos sentidos. Revista Brasileira de Educação Física e Esporte, São Paulo, v. 22, n. 2, p.119-127, abr./jun. 2008

MARINHO, A. Lazer, aventura e risco: reflexões sobre atividades realizadas na natureza. Movimento, Porto Alegre, v. 14, n. 2, p. 181-206, maio/ago. 2008.

MARKLAND, D.; TOBIN, V. A modification to the behavioural regulation in exercise questionnaire to include an assessment of amotivation. Journal of Sport and Exercise Psychology, v. 26, n. 2, p. 191-196, 2004.

MATIAS, T. S. et al. Autodeterminação de adolescentes em diferentes estágios de mudança para o exercício físico. Revista Educação Física, UEM, v. 25, n. 2, p.211-222, maio 2014. 
MATIAS, T. S. et al. The role of motivation on the associations between physical activity choices, depression symptoms and mood profile of adolescente students. 2016. $141 \mathrm{f}$. Tese (Doutorado em Ciências do Movimento Humano) - Programa de Pós-graduação em Ciências do Movimento Humano, Centro de Ciências da Saúde e do Esporte, Universidade do Estado de Santa Catarina, Florianópolis, 2016.

MORAES, L. C.; OLIVEIRA, D. C. de. Emoções em situações de risco no alpinismo de alto nível. Revista Brasileira de Psicologia do Esporte e do Exercício, Belo Horizonte, v. 0, p. 4-21, 2006.

PEREIRA, D.; ARMBRUST, I.; RICARDO, D. P. Esportes radicais, de aventura e ação: conceitos, classificações e características. Revista Corpoconsciência, Santo André, v. 12, n. 1, p.18-34, jun. 2008.

SILVA, R. B. et al. Relação da prática de exercícios físicos e fatores associados às regulações motivacionais de adolescentes brasileiros. Motricidade, [s.1.], v. 8, n. 2, p.8-21, jun., 2012.

SPINK, M. J.; ARAGAKI, S. S.; ALVES, M. P. Da exacerbação dos sentidos no encontro com a natureza: contrastando esportes radicais e turismo de aventura. Psicologia: reflexão e crítica, São Paulo, v. 18, n. 1, p.26-38, mar. 2004.

VIEIRA, L. F. et al. Estado de fluxo em praticantes de escalada e skate downhill. Motriz, Rio Claro, v. 17, n. 4, p. 591-599, dez. 2011.

VIERLING, K. K.; STANDAGE, M.; TREASURE, D. C. Predicting atitudes and physical activity in an "at-risk" minority youth sample: a testof self-determination theory. Psychology of Sport and Exercise, Philadelphia, v. 8, n. 5, p. 795-817, 2007.

Recebido em: 28/11/2017

Revisado em: 17/09/2018

Aprovado em: 24/09/2018

Endereço para correspondência:

thiagosousamatias@gmail.com

Thiago Sousa Matias

Universidade Federal de Santa Catarina

Departamento de Educação Física-DEF/CDS/UFSC

Campus Universitário, Trindade 88040-900, Florianópolis, SC 